\title{
RANDOM AFFINE CODE TREE FRACTALS: HAUSDORFF AND AFFINITY DIMENSIONS AND PRESSURE
}

\author{
ESA JÄRVENPÄÄ, MAARIT JÄRVENPÄÄ, MENG WU, AND WEN WU
}

\begin{abstract}
We prove that for random affine code tree fractals the affinity dimension is almost surely equal to the unique zero of the pressure function. As a consequence, we show that the Hausdorff, packing and box counting dimensions of such systems are equal to the zero of the pressure. In particular, we do not presume the validity of the Falconer-Sloan condition or any other additional assumptions which have been essential in all the previously known results.
\end{abstract}

\section{INTRODUCTION}

The investigation of dimensional properties of self-affine sets dates back to the pioneering works of Bedford [3] and McMullen [24]. Besides considering specific self-affine sets as in [3, 24], a natural approach is to seek generic dimension formulae. In [5], Falconer obtained a dimension formula for generic self-affine sets in terms of the pressure function under the assumption that the norms of the linear parts of generating functions are less than $1 / 3$. Later, Solomyak [29] observed that $1 / 3$ can be replaced by $1 / 2$ which, in turn, is the best possible bound due to an example given by Przytycki and Urbański [25]. Since the seminal works in [3, 5, 24], there has been great interest in various problems related to dimensions of self-affine sets. For recent contributions in this field, see for example [2, 4, 6 9, 13, 20, 27, 28] and the references therein.

We address the problem of studying a general class of random affine code tree fractals introduced in [21]. Typically, code tree fractals are locally random but globally nearly homogeneous mimicking deterministic systems. As explained in [21, 22], our setup includes several random models, for example, homogeneous graph directed systems [23] and $V$-variable fractals [1].

In [5], Falconer proved that the Hausdorff dimension of the attractor of an affine iterated function system is equal to the zero of the pressure for almost all translation vectors, and well-known examples show that this is genuinely an almost all type of result. Using Carathéodory's construction with weights determined by the singular value function, he introduced a parametrised family of net measures which behaves

2010 Mathematics Subject Classification. 37C45, 28A80, 15A45.

Key words and phrases. Hausdorff dimension, affinity dimension, self-affine sets, pressure.

We acknowledge the support of Academy of Finland, the Centre of Excellence in Analysis and Dynamics Research. Wen Wu was also supported by NSFC (Grant Nos. 11401188). 
like the Hausdorff measure in the sense that there exists a unique parameter value where the measure drops from infinity to zero. In what follows, this unique value is called the affinity dimension. According to [5], the affinity dimension equals the Hausdorff dimension for almost all translation vectors and, moreover, the affinity dimension is equal to the unique zero of the pressure, which always exists for selfaffine sets.

As far as the extendability of Falconer's result for code tree fractals is concerned, in [21] it is proved that, for all code tree fractals, the affinity dimension equals the Hausdorff dimension for almost all translation vectors (see also Theorem 2.3). However, the Hausdorff or affinity dimension need not to be equal to the zero of the pressure. In particular, the pressure does not necessarily exist. To avoid this kind of irregular behaviour, a general class of random code tree fractals with a neck structure was introduced in [21. In this class, the pressure exists and has a unique zero almost surely with respect to any shift invariant ergodic probability measure for which the expectation of the length of the first neck is finite (see [21] or Theorem 2.1). The problem whether the zero of the pressure is equal to the Hausdorff dimension was addressed in [21]. It turned out that in the plane under several additional assumptions this is indeed the case (see [21, Theorem 5.1]).

In many questions involving the pressure of affine systems, the fact that the singular value function is submultiplicative but not multiplicative causes problems (see, for example, [7, 10 12]). In our context, the Falconer-Sloan condition, introduced in [10], is useful for the purpose of overcoming these problems. Indeed, in [22] it is verified that in the $d$-dimensional case the Hausdorff dimension of a typical code tree fractal equals the zero of the pressure under a weak probabilistic version of the Falconer-Sloan condition ( [22, Theorem 3.2]). As pointed out in [22], the FalconerSloan condition, or another corresponding assumption, is necessary for the method of proof in [22]. The question whether the result could be true without additional assumptions remained open. In this paper, we answer this question by proving that this is indeed the case. Our main theorem states that for typical code tree fractals the Hausdorff, packing and box counting dimensions are equal to the zero of the pressure (see Theorem 3.5).

Our methods are completely different from those of [21,22. We introduce a new concept of a neck net measure (see Section 3) resembling the net measure that is utilised in the definition of the affinity dimension - the essential difference being that the neck net measure takes into account the neck structure. It turns out that the affinity dimension is almost surely equal to the neck affinity dimension (Proposition 3.2) which, in turn, equals the zero of the pressure (Theorem 3.5). The main part of the proof is to show that the neck affinity dimension is not smaller than the zero of the pressure. The proof of this fact is based on a careful decomposition of trees, appearing in the definitions of the pressure and the neck affinity dimension, into suitable subtrees. 
The paper is organised as follows. In Section 2, we recall the notation from [21,22] and present results needed for proving our main theorem. In Section 3, we state and prove the main result.

\section{Notation AND PRELIMINARIES}

In this section, we summon notation and preliminaries from [21]. (Note that, for simplicity, our notation is slightly different from that of [21.) Let $\Lambda$ be a topological space. Suppose that $\mathcal{F}=\left\{F^{\lambda}=\left\{T_{i}^{\lambda}+a_{i}^{\lambda}\right\}_{i=1}^{M_{\lambda}} \mid \lambda \in \Lambda\right\}$ is a family of iterated function systems on $\mathbb{R}^{d}$ consisting of affine maps. Here $T_{i}^{\lambda}: \mathbb{R}^{d} \rightarrow \mathbb{R}^{d}$ is a non-singular linear mapping and $a_{i}^{\lambda} \in \mathbb{R}^{d}$. For brevity, we write $f_{i}^{\lambda}=T_{i}^{\lambda}+a_{i}^{\lambda}$ for all $i=1, \ldots, M_{\lambda}$ and $\lambda \in \Lambda$. We assume that

$$
\sup _{\lambda \in \Lambda, i=1, \ldots, M_{\lambda}}\left|a_{i}^{\lambda}\right|<\infty
$$

and, moreover, there exist $M>0$ and $\underline{\sigma}, \bar{\sigma} \in(0,1)$ such that

$$
M=\sup _{\lambda \in \Lambda} M_{\lambda}<\infty
$$

and

$$
\underline{\sigma} \leq \sigma_{d}\left(T_{i}^{\lambda}\right) \leq \cdots \leq \sigma_{1}\left(T_{i}^{\lambda}\right)=\left\|T_{i}^{\lambda}\right\| \leq \bar{\sigma} \text { for all } \lambda \in \Lambda \text { and } i=1, \ldots, M_{\lambda}
$$

where $\sigma_{j}(T)$ is the $j$-th singular value of a non-singular linear mapping $T: \mathbb{R}^{d} \rightarrow \mathbb{R}^{d}$. Note that $F^{\lambda}$ may be naturally identified with an element of $\mathbb{R}^{\left(d^{2}+d\right) M_{\lambda}}$ and, thus, $\mathcal{F} \subset \bigcup_{i=1}^{M} \mathbb{R}^{\left(d^{2}+d\right) i}$, where the disjoint union is endowed with the natural topology. We suppose that the map $\lambda \mapsto F^{\lambda}$ is Borel measurable. For $s \geq 0$, we denote by $\Phi^{s}(T)$ the multiplicative singular value function of a linear map $T: \mathbb{R}^{d} \rightarrow \mathbb{R}^{d}$, that is,

$$
\Phi^{s}(T)= \begin{cases}\sigma_{1}(T) \sigma_{2}(T) \cdots \sigma_{m-1}(T) \sigma_{m}(T)^{s-m+1}, & \text { if } 0 \leq s \leq d \\ \sigma_{1}(T) \sigma_{2}(T) \cdots \sigma_{d-1}(T) \sigma_{d}(T)^{s-d+1}, & \text { if } s>d\end{cases}
$$

where $m$ is the integer such that $m-1 \leq s<m$.

Let $I=\{1, \ldots, M\}$ and $I^{0}=\{\emptyset\}$. For all $k \in \mathbb{N}$, the length of a word $\tau \in I^{k}$ is $|\tau|=k$. We associate to a function $\tilde{\omega}: \bigcup_{k=0}^{\infty} I^{k} \rightarrow \Lambda$ a tree rooted at $\emptyset$ in a natural manner: Let $\Sigma_{*}^{\tilde{\omega}} \subset \bigcup_{k=0}^{\infty} I^{k}$ be the unique set such that

- $\emptyset \in \Sigma_{*}^{\tilde{\omega}}$,

- if $i_{1} \cdots i_{k} \in \sum_{*}^{\tilde{\omega}}$ and $\tilde{\omega}\left(i_{1} \cdots i_{k}\right)=\lambda$, then $i_{1} \cdots i_{k} l \in \Sigma_{*}^{\tilde{\omega}}$ if and only if $l \leq M_{\lambda}$,

- if $i_{1} \cdots i_{k} \notin \Sigma_{*}^{\tilde{\omega}}$, then for all $l=1, \ldots, M$, we have $i_{1} \cdots i_{k} l \notin \Sigma_{*}^{\tilde{\omega}}$.

The restriction of $\tilde{\omega}$ to $\Sigma_{*}^{\tilde{\omega}}$ is called a code tree. In a code tree, we identify the vertex $i_{1} \cdots i_{k}$ with the function system $F^{\tilde{\omega}\left(i_{1} \cdots i_{k}\right)}$ and, moreover, the edge connecting $i_{1} \cdots i_{k}$ to $i_{1} \cdots i_{k} l$ with the map $f_{l}^{\tilde{\omega}\left(i_{1} \cdots i_{k}\right)}$. Let $\widetilde{\Omega}$ be the set of all code trees. $A$ sub code tree of a code tree $\tilde{\omega}$ is the restriction of $\tilde{\omega}$ to a subset of $\Sigma_{*}^{\tilde{\omega}}$ which is rooted at 
some vertex $i_{1} \cdots i_{k} \in \Sigma_{*}^{\tilde{\omega}}$ and contains all descendants of $i_{1} \cdots i_{k}$ belonging to $\Sigma_{*}^{\tilde{\omega}}$. We endow $\widetilde{\Omega}$ with the topology generated by the sets

$$
\left\{\tilde{\omega} \in \widetilde{\Omega} \mid \Sigma_{*}^{\tilde{\omega}} \cap \bigcup_{j=0}^{k} I^{j}=J \text { and } \tilde{\omega}(\mathbf{i}) \in U_{\mathbf{i}} \text { for all } \mathbf{i} \in J\right\},
$$

where $k \in \mathbb{N}, U_{\mathbf{i}} \subset \Lambda$ is open for all $\mathbf{i} \in J$ and $J \subset \bigcup_{j=0}^{k} I^{j}$ is a tree rooted at $\emptyset$ and having all leaves in $I^{k}$.

Equip $I^{\mathbb{N}}$ with the product topology, and define for all $\tilde{\omega} \in \widetilde{\Omega}$

$$
\Sigma^{\tilde{\omega}}=\left\{\mathbf{i}=i_{1} i_{2} \cdots \in I^{\mathbb{N}} \mid i_{1} \cdots i_{n} \in \Sigma_{*}^{\tilde{\omega}} \text { for all } n \in \mathbb{N}\right\} .
$$

Then $\Sigma^{\tilde{\omega}}$ is compact. For all $k \in \mathbb{N}$ and $\mathbf{i} \in \Sigma^{\tilde{\omega}} \cup \bigcup_{j=k}^{\infty} I^{j}$, we denote by $\mathbf{i}_{k}=i_{1} \cdots i_{k}$ the initial word of $\mathbf{i}$ with length $k$ and use following type of natural abbreviations for compositions:

$$
f_{\mathbf{i}_{k}}^{\tilde{\omega}}=f_{i_{1}}^{\tilde{\omega}(\emptyset)} \circ f_{i_{2}}^{\tilde{\omega}\left(i_{1}\right)} \circ \cdots \circ f_{i_{k}}^{\tilde{\omega}\left(i_{1} \cdots i_{k-1}\right)} \text { and } T_{\mathbf{i}_{k}}^{\tilde{\omega}}=T_{i_{1}}^{\tilde{\omega}(\emptyset)} T_{i_{2}}^{\tilde{\omega}\left(i_{1}\right)} \cdots T_{i_{k}}^{\tilde{\omega}\left(i_{1} \cdots i_{k-1}\right)} .
$$

Note that the maps $\tilde{\omega} \mapsto f_{\mathbf{i}_{k}}^{\tilde{\omega}}$ and $\tilde{\omega} \mapsto T_{\mathbf{i}_{k}}^{\tilde{\omega}}$ are Borel measurable. For all $\tilde{\omega} \in \widetilde{\Omega}$, set

$$
Z^{\tilde{\omega}}(\mathbf{i})=\lim _{k \rightarrow \infty} f_{\mathbf{i}_{k}}^{\tilde{\omega}}(0) \text { and } A^{\tilde{\omega}}=\left\{Z^{\tilde{\omega}}(\mathbf{i}) \mid \mathbf{i} \in \Sigma^{\tilde{\omega}}\right\}
$$

The attractor $A^{\tilde{\omega}}$ is called the code tree fractal corresponding to $\tilde{\omega}$. For $k \in \mathbb{N}$, $\tilde{\omega} \in \widetilde{\Omega}$ and $\mathbf{i} \in \Sigma^{\tilde{\omega}}$, the cylinder of length $k$ determined by $\mathbf{i}$ is defined as

$$
\left[\mathbf{i}_{k}\right]=\left\{\mathbf{j} \in \Sigma^{\tilde{\omega}} \mid j_{l}=i_{l} \text { for all } l=1, \ldots, k\right\} .
$$

We proceed by recalling the definition of a neck level which is an essential feature of $V$-variable fractals, see for example [1]. A neck list $\mathbf{N}=\left(N_{m}\right)_{m \in \mathbb{N}}$ is a strictly increasing sequence of natural numbers. We use the notation $\Omega$ for a subset of $\widetilde{\Omega} \times \mathbb{N}^{\mathbb{N}}$ consisting of elements $\omega=(\tilde{\omega}, \mathbf{N})$ such that

- $\mathbf{N}=\left(N_{m}\right)_{m \in \mathbb{N}}$ is a neck list and

- if $\mathbf{i}_{N_{m}} \mathbf{j}_{l}, \mathbf{i}_{N_{m}}^{\prime} \in \Sigma_{*}^{\tilde{\omega}}$, then $\mathbf{i}_{N_{m}}^{\prime} \mathbf{j}_{l} \in \sum_{*}^{\tilde{\omega}}$ and $\tilde{\omega}\left(\mathbf{i}_{N_{m}} \mathbf{j}_{l}\right)=\tilde{\omega}\left(\mathbf{i}_{N_{m}}^{\prime} \mathbf{j}_{l}\right)$.

Neck levels guarantee that the attractor $A^{\tilde{\omega}}$ is globally nearly homogeneous in the sense that if $N_{m} \in \mathbb{N}$ is a neck level of $\tilde{\omega}$, then all sub code trees of $\tilde{\omega}$ rooted at vertices $\mathbf{i} \in \Sigma_{*}^{\tilde{\omega}}$ with $|\mathbf{i}|=N_{m}$ are identical.

A function $\Xi: \Omega \rightarrow \Omega$ is a shift if $\Xi(\tilde{\omega}, \mathbf{N})=(\hat{\omega}, \hat{\mathbf{N}})$, where $\hat{N}_{m}=N_{m+1}-N_{1}$ for all $m \in \mathbb{N}$ and $\hat{\omega}\left(\mathbf{j}_{l}\right)=\tilde{\omega}\left(\mathbf{i}_{N_{1}} \mathbf{j}_{l}\right)$ for all $\mathbf{j}_{l}$ such that $\mathbf{i}_{N_{1}} \mathbf{j}_{l} \in \Sigma_{*}^{\tilde{\omega}}$. Note that, by the definition of a neck, the definition of $\hat{\omega}$ does not depend on the choice of $\mathbf{i}_{N_{1}}$. For all $i \in \mathbb{N}$ and $\omega=(\tilde{\omega}, \mathbf{N}) \in \Omega$, we write $N_{i}(\omega)=N_{i}$ for the projection of $\omega$ onto the $i$-th coordinate of $\mathbf{N}$. We equip $\Omega$ with the topology generated by cylinders

$$
\begin{gathered}
{\left[(\tilde{\omega}, \mathbf{N})_{m}\right]=\left\{(\hat{\omega}, \hat{\mathbf{N}}) \in \Omega \mid \hat{N}_{i}=N_{i} \text { for all } i \leq m \text { and } \hat{\omega}(\tau)=\tilde{\omega}(\tau)\right.} \\
\text { for all } \left.\tau \text { with }|\tau|<N_{m}\right\}
\end{gathered}
$$


Since $\omega \mapsto N_{1}(\omega)$ is continuous as a projection, the function $\omega \mapsto N_{1}(\omega)$ is Borel measurable. For any function $\phi$ of $\tilde{\omega}$, we use the notation $\phi(\omega)$ to view $\phi$ as a function of $\omega$. Finally, for all $n, m \in \mathbb{N} \cup\{0\}$ with $n<m$, define

$$
\Sigma_{*}^{\omega}(n, m)=\left\{i_{N_{n}(\omega)+1} \cdots i_{N_{m}(\omega)} \mid \mathbf{i}_{N_{n}(\omega)} i_{N_{n}(\omega)+1} \cdots i_{N_{m}(\omega)} \in \Sigma_{*}^{\omega}\right\},
$$

where $N_{0}=0$. For all $s \geq 0$, the pressure is defined as follows

$$
p^{\omega}(s)=\lim _{k \rightarrow \infty} \frac{\log S^{\omega}(k, s)}{k}
$$

provided that the limit exists. Here

$$
S^{\omega}(k, s)=\sum_{\mathbf{i}_{k} \in \Sigma_{*}^{\omega}} \Phi^{s}\left(T_{\mathbf{i}_{k}}^{\omega}\right)
$$

for all $k \in \mathbb{N}$. Since $T \mapsto \Phi^{s}(T)$ is a continuous function, the map $\omega \mapsto p^{\omega}(s)$ is Borel measurable.

It is well known that for affine iterated function systems the pressure always exists. For code tree fractals this is not always the case as explained in [21]. However, for typical random code tree fractals the pressure function exists and has a unique zero (see [21, Theorem 4.3]).

Theorem 2.1. Assume that $P$ is an ergodic $\Xi$-invariant Borel probability measure on $\Omega$ such that $\int_{\Omega} N_{1}(\omega) d P(\omega)<\infty$. Then for P-almost all $\omega \in \Omega$, the pressure $p^{\omega}(s)$ exists for all $s \in\left[0, \infty\left[\right.\right.$. Furthermore, $p^{\omega}$ is strictly decreasing and there exists a unique $s_{0}$ such that $p^{\omega}\left(s_{0}\right)=0$ for P-almost all $\omega \in \Omega$.

For the purpose of identifying certain translation vectors (for motivation of the identification, we refer to [21, 22] $)$, we equip the set $\widehat{\Lambda}=\{(\lambda, i) \mid \lambda \in \Lambda$ and $i=$ $\left.1, \ldots, M_{\lambda}\right\}$ with an equivalence relation $\sim$ satisfying the following assumptions:

- the cardinality $\mathcal{A}$ of the set of equivalence classes $\mathbf{a}:=\widehat{\Lambda} / \sim$ is finite,

- for every $\lambda \in \Lambda$, we have $(\lambda, i) \sim(\lambda, j)$ if and only if $i=j$ and

- the equivalence classes, regarded as subsets of $\Lambda$, are Borel sets.

Using the relation $\sim$ for the purpose of identifying translation vectors, the set of equivalence classes a may be viewed as an element of $\mathbb{R}^{d \mathcal{A}}$. We write $A_{\mathbf{a}}^{\omega}$ for the attractor of a code tree $\omega$ to emphasise the dependence on $\mathbf{a}$.

For determining the almost sure value of Hausdorff dimension for random affine code tree fractals, the affinity dimension turns out to be useful. The affinity dimension is defined in terms of the $s$-dimensional net measure in the following manner. Let $s \geq 0$. We denote by $\mathcal{M}^{s}$ the $s$-dimensional net measure defined for all Borel sets $E \subset \Sigma^{\omega}$ by

where

$$
\mathcal{M}^{s}(E)=\lim _{j \rightarrow \infty} \mathcal{M}_{j}^{s}(E)
$$

$$
\mathcal{M}_{j}^{s}(E)=\inf \left\{\sum_{\mathbf{i}_{k} \in J} \Phi^{s}\left(T_{\mathbf{i}_{k}}^{\omega}\right) \mid J \subset \Sigma_{*}^{\omega}, E \subset \bigcup_{\mathbf{i}_{k} \in J}\left[\mathbf{i}_{k}\right] \text { and } k \geq j\right\} .
$$


The affinity dimension of $\Sigma^{\omega}$ is defined as

$$
\alpha^{\omega}=\inf \left\{s \geq 0 \mid \mathcal{M}^{s}\left(\Sigma^{\omega}\right)=0\right\}=\sup \left\{s \geq 0 \mid \mathcal{M}^{s}\left(\Sigma^{\omega}\right)=\infty\right\} .
$$

Remark 2.2. According to the standard definition, the affinity dimension is the unique zero of the pressure determined by the singular value function. Note that in the case when the maps are similarities, the zero of the pressure equals the similarity dimension of a self-similar set. In [5], Falconer proved that, for affine iterated function systems, the pressure exists and its unique zero equals the number $\alpha^{\omega}$ defined in (2.5). As pointed out in the introduction, for general code tree fractals the pressure need not exist and even if it exists, it is not necessarily equal to the number $\alpha^{\omega}$ (see [21]). Since $\alpha^{\omega}$ is defined for any code tree fractal, we prefer it as the affinity dimension. Our choice is strongly supported by the following theorem.

We denote the Hausdorff dimension by $\operatorname{dim}_{\mathrm{H}}$. According to [21, Theorem 3.2], for all $\omega \in \Omega$, Hausdorff and affinity dimensions of $A_{\mathbf{a}}^{\omega}$ agree for almost all $\mathbf{a}$.

Theorem 2.3. Assume that $\bar{\sigma}<1 / 2$. Let $\omega \in \Omega$. Then

$$
\operatorname{dim}_{\mathrm{H}} A_{\mathbf{a}}^{\omega}=\min \left\{\alpha^{\omega}, d\right\} \text { for } \mathcal{L}^{d \mathcal{A}} \text {-almost all } \mathbf{a} \in \mathbb{R}^{d \mathcal{A}} .
$$

\section{Results}

In this section, we state and prove our main result (Theorem 3.5), according to which, almost surely with respect to any ergodic $\Xi$-invariant measure having finite expectation for the first neck level, the Hausdorff, packing and box counting dimensions of code tree fractals are equal to the zero of the pressure for almost all translation vectors.

We proceed by verifying auxiliary results. Our first aim is to prove that the affinity dimension $\alpha^{\omega}$ is constant almost surely.

Proposition 3.1. Let $P$ be an ergodic $\Xi$-invariant probability measure on $\Omega$ with $\int_{\Omega} N_{1}(\omega) d P(\omega)<\infty$. There exists $\alpha \geq 0$ such that $\alpha^{\omega}=\alpha$ for P-almost all $\omega \in \Omega$.

Proof. Since $\int_{\Omega} N_{1}(\omega) d P(\omega)<\infty$, we have $N_{1}(\omega)<\infty$ for $P$-almost all $\omega \in \Omega$. For all such $\omega \in \Omega$, the definition of $\mathcal{M}_{n}^{s}$ implies that

$$
\left(\underline{\sigma}^{s}\right)^{N_{1}(\omega)} \mathcal{M}_{n}^{s}\left(\Sigma^{\Xi(\omega)}\right) \leq \mathcal{M}_{N_{1}(\omega)+n}^{s}\left(\Sigma^{\omega}\right) \leq\left(M \bar{\sigma}^{s}\right)^{N_{1}(\omega)} \mathcal{M}_{n}^{s}\left(\Sigma^{\Xi(\omega)}\right)
$$

for all $s \geq 0$ and $n \in \mathbb{N}$. Letting $n \rightarrow \infty$, gives

$$
\left(\underline{\sigma}^{s}\right)^{N_{1}(\omega)} \mathcal{M}^{s}\left(\Sigma^{\Xi(\omega)}\right) \leq \mathcal{M}^{s}\left(\Sigma^{\omega}\right) \leq\left(M \bar{\sigma}^{s}\right)^{N_{1}(\omega)} \mathcal{M}^{s}\left(\Sigma^{\Xi(\omega)}\right) .
$$

From (3.1), we deduce that the set

$$
E(s):=\left\{\omega \in \Omega \mid \mathcal{M}^{s}\left(\Sigma^{\omega}\right)>0\right\}
$$

is $\Xi$-invariant, that is, $\Xi^{-1}\left((E(s))=E(s)\right.$. Since $\Sigma^{\omega}$ is compact and cylinder sets are open, one may use finite coverings when calculating $\mathcal{M}_{j}^{s}\left(\Sigma^{\omega}\right)$. Thus, the Borel measurability of $\omega \mapsto \Phi^{s}\left(T_{\mathbf{i}_{k}}^{\omega}\right)$ implies that $\omega \mapsto \mathcal{M}^{s}\left(\Sigma^{\omega}\right)$ is a Borel map and, therefore, $E(s)$ is a Borel set. Since $P$ is ergodic, for all $s \geq 0, P(E(s))$ is either 0 or 1 . 
It follows that $\alpha^{\omega}$ is a constant for $P$-almost all $\omega \in \Omega$, since otherwise there exists $s \geq 0$ such that $0<P(E(s))<1$.

Now we introduce another net measure $\widetilde{\mathcal{M}}^{s}$ on $\Sigma^{\omega}$ which is similar to the natural net measure $\mathcal{M}^{s}$ but takes into account the neck structure. For all $n \in \mathbb{N}$, let

$$
I_{n}=\left\{\mathbf{i}_{N_{k}(\omega)} \in \Sigma_{*}^{\omega} \mid k \geq n\right\} .
$$

For $s \geq 0$, the $s$-dimensional neck net measure $\widetilde{\mathcal{M}^{s}}$ is defined for all Borel subsets $E$ of $\Sigma^{\omega}$ by

$$
\widetilde{\mathcal{M}}^{s}(E)=\lim _{j \rightarrow \infty} \widetilde{\mathcal{M}}_{j}^{s}(E)
$$

where

$$
\widetilde{\mathcal{M}}_{j}^{s}(E)=\inf \left\{\sum_{\mathbf{i} \in I} \Phi^{s}\left(T_{\mathbf{i}}^{\omega}\right) \mid E \subset \bigcup_{\mathbf{i} \in I}[\mathbf{i}] \text { and } I \subset I_{j}\right\} .
$$

We define the neck affinity dimension of $\Sigma^{\omega}$ as

$$
\tilde{\alpha}^{\omega}=\inf \left\{s \geq 0 \mid \widetilde{\mathcal{M}}^{s}\left(\Sigma^{\omega}\right)=0\right\}=\sup \left\{s \geq 0 \mid \widetilde{\mathcal{M}}^{s}\left(\Sigma^{\omega}\right)=\infty\right\} .
$$

Since in the definition of $\widetilde{\mathcal{M}}^{s}(E)$ there are more restrictions on possible coverings of $E$ than in the case of $\mathcal{M}^{s}(E)$, we have $\mathcal{M}^{s}(E) \leq \widetilde{\mathcal{M}}^{s}(E)$. Hence,

$$
\alpha^{\omega} \leq \tilde{\alpha}^{\omega} \text { for all } \omega \in \Omega \text {. }
$$

It turns out that $\alpha^{\omega}$ and $\tilde{\alpha}^{\omega}$ are equal almost surely.

Proposition 3.2. Letting $P$ be an ergodic $\Xi$-invariant probability measure on $\Omega$ with $\int_{\Omega} N_{1}(\omega) d P(\omega)<\infty$, we have that $\alpha^{\omega}=\tilde{\alpha}^{\omega}$ for P-almost all $\omega \in \Omega$.

Proof. Observe that there exists a Borel set $F \subset \Omega$ with $P(F)=1$ such that for all $\omega \in F$ and $\delta>0$, we have

$$
N_{n+1}(\omega)-N_{n}(\omega) \leq \delta N_{n}(\omega)
$$

for sufficiently large $n \in \mathbb{N}$. Indeed, since

$$
N_{n}(\omega)=\sum_{k=0}^{n-1}\left(N_{k+1}(\omega)-N_{k}(\omega)\right)=\sum_{k=0}^{n-1} N_{1}\left(\Xi^{k}(\omega)\right),
$$

Birkhoff's ergodic theorem gives

$$
\lim _{n \rightarrow \infty} \frac{N_{n}(\omega)}{n}=\lim _{n \rightarrow \infty} \frac{1}{n} \sum_{k=0}^{n-1} N_{1}\left(\Xi^{k}(\omega)\right)=\int_{\Omega} N_{1}(\omega) d P(\omega)
$$

for $P$-almost all $\omega \in \Omega$. This leads to

$$
\lim _{n \rightarrow \infty} \frac{N_{n+1}(\omega)-N_{n}(\omega)}{n}=0=\lim _{n \rightarrow \infty} \frac{N_{n+1}(\omega)-N_{n}(\omega)}{N_{n}(\omega)}
$$

for $P$-almost all $\omega \in \Omega$, completing the proof of (3.4). 
Now, by (3.3), it suffices to verity that

$$
\alpha^{\omega} \geq \tilde{\alpha}^{\omega} \text { for } P \text {-almost all } \omega \in F .
$$

For this purpose, consider $\omega \in F$ and $\varepsilon>0$. We will show that there exist a finite Borel measure $\mu^{\omega}$ on $\Sigma^{\omega}$ and a constant $c(\omega)$ such that

$$
\mu^{\omega}\left[\mathbf{i}_{k}\right] \leq c(\omega) \Phi^{\tilde{\alpha}^{\omega}-\varepsilon}\left(T_{\mathbf{i}_{k}}^{\omega}\right)
$$

for all cylinders $\left[\mathbf{i}_{k}\right]$ with $k \geq 1$. It is shown in the proof of [21, Theorem 3.2] that this results in

$$
\operatorname{dim}_{\mathrm{H}} A_{\mathbf{a}}^{\omega} \geq \tilde{\alpha}^{\omega}-\varepsilon \text { for } \mathcal{L}^{d \mathcal{A}} \text {-almost all } \mathbf{a} \in \mathbb{R}^{d \mathcal{A}} .
$$

From Theorem 2.3, we conclude that $\alpha^{\omega} \geq \tilde{\alpha}^{\omega}-\varepsilon$ for $P$-almost all $\omega \in F$. Taking a sequence $\varepsilon_{i}$ tending to zero, completes the proof of (3.6).

It remains to prove (3.7). Since $\widetilde{\mathcal{M}}^{\tilde{\alpha}^{\omega}-\varepsilon / 2}\left(\Sigma^{\omega}\right)=\infty$, we derive, similarly as in [26, Proposition 2.8] (the proof of [26, Proposition 2.8] is written for homogeneous code trees but it works for inhomogeneous code trees as well), that there exist a finite Borel measure $\mu^{\omega}$ on $\Sigma^{\omega}$ and a constant $c^{\prime}(\omega)$ such that

$$
\mu^{\omega}\left[\mathbf{i}_{N_{k}(\omega)}\right] \leq c^{\prime}(\omega) \Phi^{\tilde{\alpha}^{\omega}-\varepsilon / 2}\left(T_{\mathbf{i}_{N_{k}}(\omega)}^{\omega}\right)
$$

for all cylinders $\left[\mathbf{i}_{N_{k}(\omega)}\right]$ with $k \geq 1$. We aim to verify that $\mu^{\omega}$ satisfies (3.7). For all $n \in \mathbb{N}$, there is a unique $k \in \mathbb{N}$ such that $N_{k}(\omega) \leq n<N_{k+1}(\omega)$. By (3.8) and the submultiplicativity of $\Phi^{s}$, we get

$$
\begin{aligned}
\mu^{\omega}\left[\mathbf{i}_{n}\right] & =\sum_{i_{n+1}, \ldots, i_{N_{k+1}(\omega)}} \mu^{\omega}\left[\mathbf{i}_{n} i_{n+1} \cdots i_{N_{k+1}(\omega)}\right] \\
& \leq c^{\prime}(\omega) \Phi^{\tilde{\alpha}^{\omega}-\varepsilon / 2}\left(T_{\mathbf{i}_{n}}^{\omega}\right) \sum_{i_{n+1}, \ldots, i_{N_{k+1}(\omega)}} \Phi^{\tilde{\alpha}^{\omega}-\varepsilon / 2}\left(T_{i_{n+1}}^{\omega} \cdots T_{i_{N_{k+1}(\omega)}}^{\omega}\right) \\
& =c^{\prime}(\omega) \Phi^{\tilde{\alpha}^{\omega}-\varepsilon}\left(T_{\mathbf{i}_{n}}^{\omega}\right) \frac{\Phi^{\tilde{\alpha}^{\omega}-\varepsilon / 2}\left(T_{\mathbf{i}_{n}}^{\omega}\right)}{\Phi^{\tilde{\alpha}^{\omega}-\varepsilon}\left(T_{\mathbf{i}_{n}}^{\omega}\right)} \sum_{i_{n+1}, \ldots, i_{N_{k+1}(\omega)}} \Phi^{\tilde{\alpha}^{\omega}-\varepsilon / 2}\left(T_{i_{n+1}}^{\omega} \cdots T_{i_{N_{k+1}(\omega)}}^{\omega}\right) .
\end{aligned}
$$

The assumptions (2.2) and (2.3) imply that

$$
a:=\sum_{i_{n+1}, \ldots, i_{N_{k+1}}(\omega)} \Phi^{\tilde{\alpha}^{\omega}-\varepsilon / 2}\left(T_{i_{n+1}}^{\omega} \cdots T_{i_{N_{k+1}}(\omega)}^{\omega}\right) \leq M^{N_{k+1}(\omega)-n} \leq M^{N_{k+1}(\omega)-N_{k}(\omega)}
$$

and

$$
b:=\frac{\Phi^{\tilde{\alpha}^{\omega}-\varepsilon / 2}\left(T_{\mathbf{i}_{n}}^{\omega}\right)}{\Phi^{\tilde{\alpha}^{\omega}-\varepsilon}\left(T_{\mathbf{i}_{n}}^{\omega}\right)} \leq \bar{\sigma}^{\varepsilon n / 2} \leq \bar{\sigma}^{\varepsilon N_{k}(\omega) / 2} .
$$

As $\omega \in F$, for every $\delta>0$, we have $N_{k+1}(\omega)-N_{k}(\omega) \leq \delta N_{k}(\omega)$ when $k$ is large enough. Since $\bar{\sigma}<1$ and $M<\infty$, taking $\delta$ sufficiently small, gives $a b \leq 1$ for all 
sufficiently large $k \in \mathbb{N}$. Substituting the product of $a$ and $b$ with 1 in (3.9), we obtain that

$$
\mu^{\omega}\left[\mathbf{i}_{n}\right] \leq c^{\prime}(\omega) \Phi^{\tilde{\alpha}^{\omega}-\varepsilon}\left(T_{\mathbf{i}_{n}}^{\omega}\right)
$$

for large enough $n \in \mathbb{N}$. Thus we can find a constant $c(\omega)$ such that

$$
\mu^{\omega}\left[\mathbf{i}_{n}\right] \leq c(\omega) \Phi^{\tilde{\alpha}^{\omega}-\varepsilon}\left(T_{\mathbf{i}_{n}}^{\omega}\right)
$$

for all cylinders $\left[\mathbf{i}_{n}\right]$ with $n \geq 1$.

When calculating $\widetilde{\mathcal{M}}^{s}\left(\Sigma^{\omega}\right)$ for $s>\tilde{\alpha}^{\omega}$, by compactness of $\Sigma^{\omega}$, one may find a finite covering $\left\{\left[\mathbf{i}_{N_{k}}\right]\right\}_{\mathbf{i}_{N_{k}} \in J}$ of $\Sigma^{\omega}$ such that $\sum_{\mathbf{i}_{N_{k}} \in J} \Phi^{s}\left(T_{\mathbf{i}_{N_{k}}}^{\omega}\right)<1$. In particular, the lengths of $\mathbf{i}_{N_{k}}$ are bounded but the bound may depend on $\omega \in \Omega$. Next we prove a lemma which states that, apart from a small exceptional set, one can find a uniform bound. In what follows we need the following notation. For all $n \in \mathbb{N}$ and $\omega \in \Omega$, set

$$
J_{n}(\omega)=\bigcup_{k=1}^{n}\left\{\mathbf{i}_{N_{k}(\omega)} \mid \mathbf{i}_{N_{k}(\omega)} \in \Sigma_{*}^{\omega}\right\} \text { and } J(\omega)=\bigcup_{k=1}^{\infty} J_{k}(\omega) .
$$

Lemma 3.3. Let $P$ be an ergodic $\Xi$-invariant probability measure on $\Omega$ satisfying $\int_{\Omega} N_{1}(\omega) d P(\omega)<\infty$, and let $s>\alpha$, where $\alpha$ is as in Proposition 3.1. For every $\varepsilon>0$, there exist a Borel set $B(\varepsilon) \subset \Omega$ with $P(B(\varepsilon)) \geq 1-\varepsilon$ and $R \in \mathbb{N}$ such that for every $\omega \in B(\varepsilon)$, there is $C_{R}(\omega) \subset J_{R}(\omega)$ with

$$
\Sigma^{\omega} \subset \bigcup_{\mathbf{i}_{N_{k}}(\omega) \in C_{R}(\omega)}\left[\mathbf{i}_{N_{k}(\omega)}\right] \text { and } \sum_{\mathbf{i}_{N_{k}(\omega)} \in C_{R}(\omega)} \Phi^{s}\left(T_{\mathbf{i}_{N_{k}}(\omega)}^{\omega}\right)<1 .
$$

Proof. We introduce a family of functions $\left\{f_{n}\right\}_{n \in \mathbb{N}}$ defined on $\Omega$ by

$$
f_{n}(\omega)=\min \left\{\sum_{\mathbf{i}_{N_{k}(\omega)} \in I} \Phi^{s}\left(T_{\mathbf{i}_{N_{k}(\omega)}}^{\omega}\right) \mid \Sigma^{\omega} \subset \bigcup_{\mathbf{i}_{N_{k}(\omega)} \in I}\left[\mathbf{i}_{N_{k}(\omega)}\right] \text { and } I \subset J_{n}(\omega)\right\} .
$$

For all $n \in \mathbb{N}$, the function $f_{n}(\omega)$ is Borel measurable. Indeed, this follows from the fact that $\omega \mapsto \sum_{\mathbf{i}_{N_{k}(\omega)} \in I} \Phi^{s}\left(T_{\mathbf{i}_{N_{k}}(\omega)}^{\omega}\right)$ is a Borel function as a finite sum of Borel functions.

Recalling that $\tilde{\alpha}^{\omega}=\alpha$ for $P$-almost all $\omega \in \Omega$ (see Propositions 3.1 and 3.2), gives $\widetilde{\mathcal{M}^{s}}\left(\Sigma^{\omega}\right)=0$ for $P$-almost all $\omega \in \Omega$. In particular, for $P$-almost all $\omega \in \Omega$ and for every $\delta>0$, there exists a finite set $I \subset J(\omega)$ such that

$$
\sum_{\mathbf{i}_{N_{k}(\omega)} \in I} \Phi^{s}\left(T_{\mathbf{i}_{N_{k}}(\omega)}^{\omega}\right)<\delta \text { and } \Sigma^{\omega} \subset \bigcup_{\mathbf{i}_{N_{k}(\omega)} \in I}\left[\mathbf{i}_{N_{k}(\omega)}\right]
$$

Recall that we may use finite coverings in the definition of $\widetilde{\mathcal{M}}^{s}\left(\Sigma^{\omega}\right)$ because $\Sigma^{\omega}$ is compact and every cylinder in $\Sigma_{*}^{\omega}$ is an open set. This implies that for $P$-almost all $\omega \in \Omega$, we have

$$
\lim _{n \rightarrow \infty} f_{n}(\omega)=0
$$


By Egorov's theorem, for every $\varepsilon>0$, there exists a Borel set $B(\varepsilon) \subset \Omega$ with $P(B(\varepsilon)) \geq 1-\varepsilon$ such that $f_{n}(\omega)$ converges uniformly to 0 on $B(\varepsilon)$. In particular, there exists $R \in \mathbb{N}$ such that $f_{R}(\omega)<1$ for all $\omega \in B(\varepsilon)$.

Our plan is to show that for any $s>\alpha$, we have $s \geq s_{0}$, that is, $p^{\omega}(s) \leq 0$. To achieve this goal, we will employ Lemma 3.3 and, for all large enough $L \in \mathbb{N}$, we decompose the finite code tree

$$
\Sigma^{\omega}(L)=\left\{\mathbf{i}_{N_{L}(\omega)} \mid \mathbf{i}_{N_{L}(\omega)} \in \Sigma_{*}^{\omega}\right\}
$$

into subtrees $C_{R}\left(\omega^{\prime}\right)$ given by Lemma 3.3 for some suitable $\omega^{\prime} \in \Omega$. Since the complement of the set $B(\varepsilon)$ in Lemma 3.3 may have positive measure, the above decomposition cannot cover the whole tree $\Sigma^{\omega}(L)$. However, the ergodicity of $P$ guarantees that the contribution of the remaining part is not too large.

Now we formalise the above idea. Consider $s>\alpha$ and $\varepsilon>0$. Let $B(\varepsilon) \subset \Omega$ and $R \in \mathbb{N}$ be as in Lemma 3.3. Set

$$
C(\varepsilon)=\left\{C_{R}(\omega) \mid \omega \in B(\varepsilon)\right\},
$$

and for all $L \in \mathbb{N}$ with $L \geq R$, define

$$
\begin{aligned}
H(\varepsilon, L)=\{ & \left\{i_{N_{k}(\omega)} \cdots i_{N_{k^{\prime}}(\omega)} \mid i_{N_{k}(\omega)} \cdots i_{N_{k^{\prime}}(\omega)} \in \Sigma_{*}^{\Xi^{k}(\omega)}\right\} \mid k<L, \Xi^{k}(\omega) \notin B(\varepsilon) \text { and } \\
& \left.k^{\prime}=\min \left\{L, \min \left\{j>k \mid \Xi^{j}(\omega) \in B(\varepsilon)\right\}\right\}\right\} .
\end{aligned}
$$

Note that each element in $C(\varepsilon)$ is a collection of words with possibly varying length at most $R$ while every element of $H(\varepsilon, L)$ is a collection of words with same length, which is at most $N_{L}(\omega)$. Each element in $C(\varepsilon)$ and $H(\varepsilon, L)$ is called a subtree. For every $\omega \in \Omega$ and $L \geq R$, we decompose $\Sigma^{\omega}(L)$ into subtrees belonging to $C(\varepsilon)$ and $H(\varepsilon, L)$. The decomposition will be done inductively as follows.

Decomposition step 1. If $\omega \in B(\varepsilon)$, then the first generation decomposition subtree $D_{1}(\omega)$ is $C_{R}(\omega)$ given by Lemma 3.3. Otherwise, let

$$
m(\omega)=\min \left\{L, \min \left\{k \geq 1 \mid \Xi^{k}(\omega) \in B(\varepsilon)\right\}\right\}
$$

and define $D_{1}(\omega)$ as $\left\{\mathbf{i}_{N_{m(\omega)}(\omega)} \mid \mathbf{i}_{N_{m(\omega)}(\omega)} \in \Sigma_{*}^{\omega}\right\} \in H(\varepsilon, L)$. In both cases, the words $\mathbf{i}_{N_{k}(\omega)} \in D_{1}(\omega)$ satisfy $k \leq L$ and, moreover, the cylinders $\left[\mathbf{i}_{N_{k}(\omega)}\right]$ are disjoint and cover the whole space $\Sigma^{\omega}$.

Decomposition step 2. We apply the decomposition step 1 to the descendants of each $\mathbf{i}_{N_{k}(\omega)} \in D_{1}(\omega)$ with $k \leq L-R$. More precisely, for every $\mathbf{i}_{N_{k}(\omega)} \in D_{1}(\omega)$ with $k \leq L-R$, we set $\omega^{\prime}=\Xi^{k}(\omega)$ and apply decomposition step 1 with $\omega$ replaced by $\omega^{\prime}$ and with the modification that in the definition of $m\left(\omega^{\prime}\right) L$ is replaced by $L-k$. In this way, every $\mathbf{i}_{N_{k}(\omega)} \in D_{1}(\omega)$ with $k \leq L-R$ defines a second generation decomposition subtree $D_{2}\left(\omega, \mathbf{i}_{N_{k}(\omega)}\right)$. We continue this decomposition process inductively until all the nodes in $\Sigma^{\omega}(L)$ which are not covered lie between levels $N_{L-R}(\omega)$ and $N_{L}(\omega)$. In this manner, we obtain a tree, denoted by $A(L)$, consisting of subtrees belonging to $C(\varepsilon)$ or $H(\varepsilon, L)$. 
Now we are ready to prove the main technical lemma of this paper. We denote the characteristic function of a set $A$ by $\mathbf{1}_{A}$ and the complement of $A$ by $A^{c}$.

Lemma 3.4. Consider $s>\alpha, \varepsilon>0$ and $\omega \in \Omega$. Let $B(\varepsilon)$ and $R \in \mathbb{N}$ be as in Lemma 3.3. For every $L \geq R$, we have

$$
\sum_{\mathbf{i}_{N_{L}(\omega)} \in \Sigma_{*}^{\omega}} \Phi^{s}\left(T_{\mathbf{i}_{N_{L}(\omega)}^{\omega}}^{\omega}\right) \leq M^{Q_{L}(\varepsilon)+N_{L}(\omega)-N_{L-R}(\omega)}
$$

where $Q_{L}(\varepsilon)=\sum_{k=0}^{L-1}\left(N_{k+1}(\omega)-N_{k}(\omega)\right) \mathbf{1}_{B(\varepsilon)^{c}}\left(\Xi^{k}(\omega)\right)$.

Proof. Recalling that in the tree $A(L)$ every word has length between $N_{L-R}(\omega)$ and $N_{L}(\omega)$ and utilising the submultiplicativity of $\Phi^{s}$ and the inequality $\bar{\sigma}<1$, it follows easily that

$$
\sum_{\mathbf{i}_{N_{L}(\omega)} \in \Sigma_{*}^{\omega}} \Phi^{s}\left(T_{\mathbf{i}_{N_{L}(\omega)}}^{\omega}\right) \leq\left(\sum_{\mathbf{i} \in A(L)} \Phi^{s}\left(T_{\mathbf{i}}^{\omega}\right)\right) M^{N_{L}(\omega)-N_{L-R}(\omega)} .
$$

Hence, we only need to show that

$$
\sum_{\mathbf{i} \in A(L)} \Phi^{s}\left(T_{\mathbf{i}}^{\omega}\right) \leq M^{Q_{L}(\varepsilon)}
$$

Let $\Gamma$ be any subtree of $A(L)$ consisting of the subtrees obtained in the decomposition process. The first and the last neck levels of $\Gamma$ are denoted by $F(\Gamma)$ and $L(\Gamma)$, respectively. See Figure 1 for an illustration of $F(\Gamma)$ and $L(\Gamma)$ of a subtree $\Gamma$. We are going to prove that

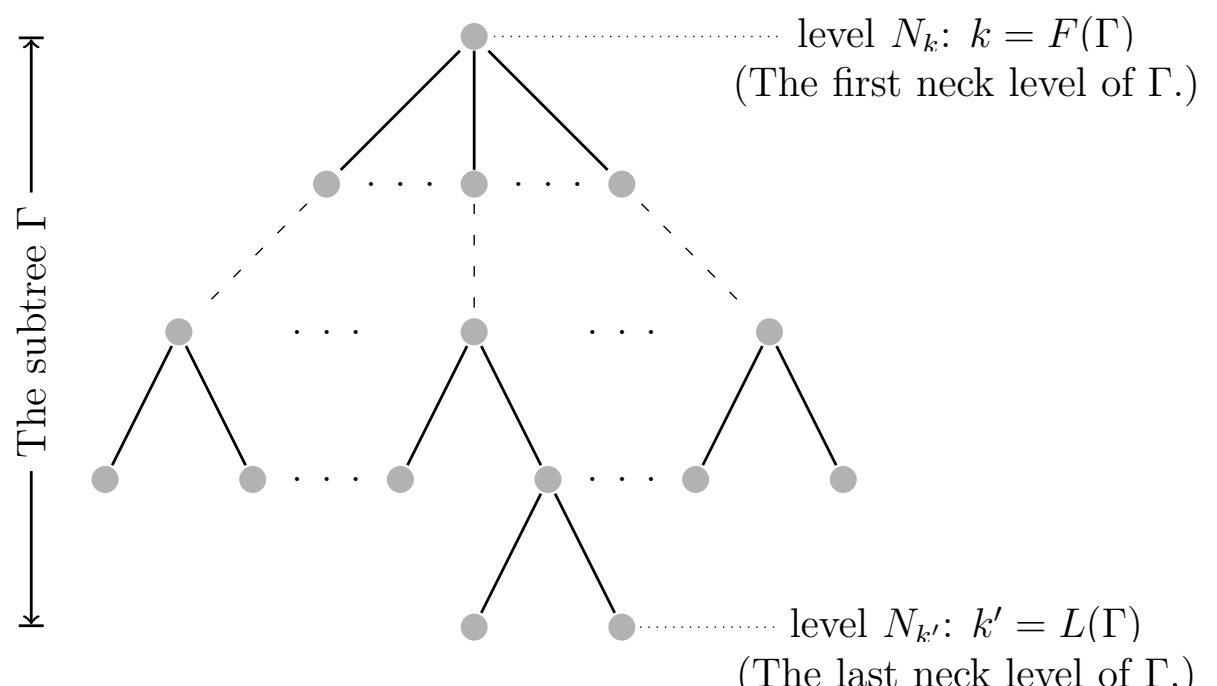

Figure 1. A subtree $\Gamma$ with its first and last neck levels. 


$$
\sum_{\mathbf{i} \in \Gamma} \Phi^{s}\left(T_{\mathbf{i}}^{\omega}\right) \leq M^{\sum_{k=F(\Gamma)}^{L(\Gamma)-1}\left(N_{k+1}(\omega)-N_{k}(\omega)\right) \mathbf{1}_{B(\varepsilon)^{c}}\left(\Xi^{k}(\omega)\right)} .
$$

Applying (3.12) to the subtree $\Gamma=A(L)$, gives (3.11). We prove (3.12) by induction on the number of the subtrees in $\Gamma$ given by the decomposition process.

Initial step: Assume that the subtree $\Gamma$ consists of one single subtree belonging to $C(\varepsilon)$ or $H(\varepsilon, L)$. If $\Gamma \in C(\varepsilon)$, the definition of $C(\varepsilon)$ results in

$$
\sum_{\mathbf{i} \in \Gamma} \Phi^{s}\left(T_{\mathbf{i}}^{\omega}\right) \leq 1 \leq M^{\sum_{k=F(\Gamma)}^{L(\Gamma)-1}\left(N_{k+1}(\omega)-N_{k}(\omega)\right) \mathbf{1}_{B(\varepsilon)^{c}}\left(\Xi^{k}(\omega)\right)} .
$$

On the other hand, if $\Gamma \in H(\varepsilon, L)$, then $\Gamma=\left\{i_{N_{k}(\omega)} \cdots i_{N_{k^{\prime}}(\omega)} \mid i_{N_{k}(\omega)} \cdots i_{N_{k^{\prime}}(\omega)} \in\right.$ $\left.\Sigma_{*}^{\Xi^{k}(\omega)}\right\}$ with $\Xi^{j}(\omega) \notin B(\varepsilon)$ for $j=k, \ldots, k^{\prime}-1$, and $\Xi^{k^{\prime}}(\omega) \in B(\varepsilon)$ or $k^{\prime}=L$. Hence,

$$
\sum_{\mathbf{i} \in \Gamma} \Phi^{s}\left(T_{\mathbf{i}}^{\omega}\right) \leq \sum_{\mathbf{i} \in \Gamma} 1 \leq M^{N_{L(\Gamma)}(\omega)-N_{F(\Gamma)}(\omega)}=M^{\sum_{k=F(\Gamma)}^{L(\Gamma)-1}\left(N_{k+1}(\omega)-N_{k}(\omega)\right) \mathbf{1}_{B(\varepsilon)^{c}}\left(\Xi^{k}(\omega)\right)} .
$$

We deduce that (3.12) holds when $\Gamma$ contains only one subtree.

Inductive step $n$ : Suppose that (3.12) is true for every subtree containing at most $n-1$ decomposition subtrees. Letting $\Gamma$ be a subtree containing $n$ decomposition subtrees, we show that (3.12) holds for $\Gamma$. For this purpose, we denote by $\Gamma(1)$ the subtree of the smallest generation in $\Gamma$. Recall that $\Gamma(1)$ belongs to $C(\varepsilon)$ or $H(\varepsilon, L)$. Suppose that $\Gamma(1)=\left\{\mathbf{i}_{1}, \mathbf{i}_{2}, \ldots, \mathbf{i}_{\gamma}\right\}$. For $\ell=1, \ldots, \gamma$, let $\Gamma_{\ell}$ be the subtree of $\Gamma$ rooted at $\mathbf{i}_{\ell}$. See Figure 2 for an illustration of $\Gamma(1)$ and $\Gamma_{\ell}$.

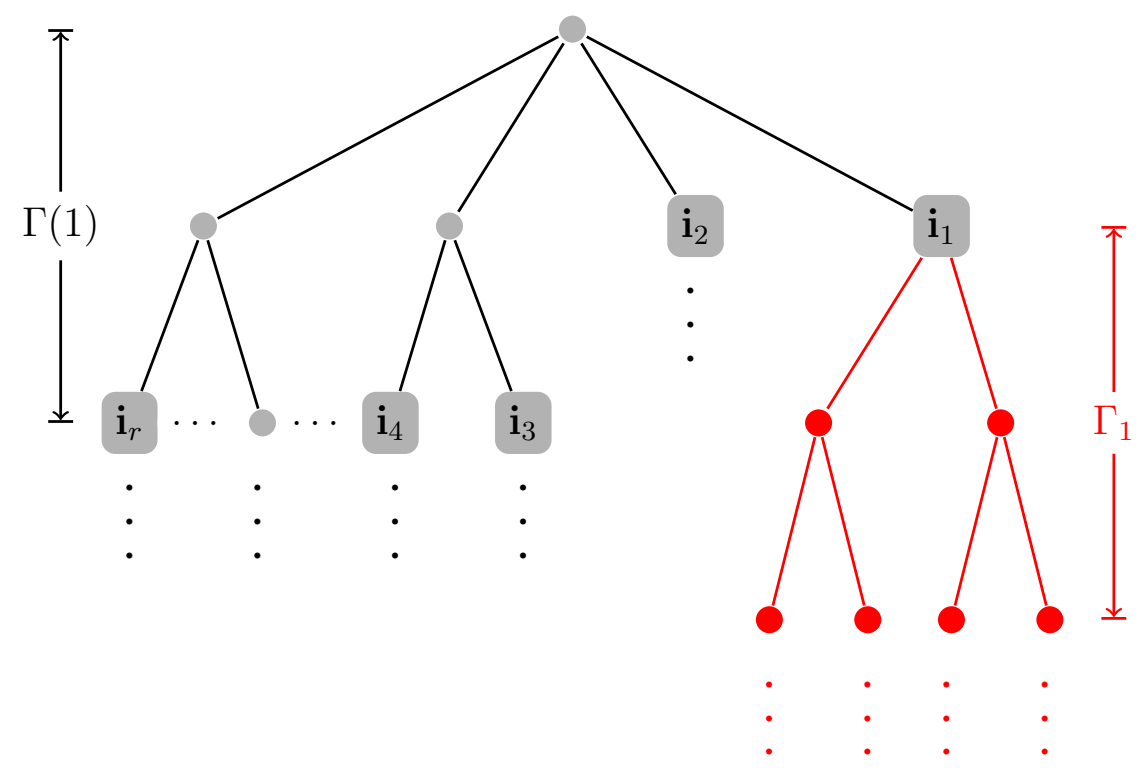

Figure 2. The first generation $\Gamma(1)$ of $\Gamma$ (in black) and the subtree $\Gamma_{1}$ rooted at $\mathbf{i}_{1}$ (in red). 
Let

$$
F\left(\Gamma_{\ell_{0}}\right)=\min _{1 \leq \ell \leq \gamma}\left\{F\left(\Gamma_{\ell}\right)\right\} \text { and } L\left(\Gamma_{\ell_{1}}\right)=\max _{1 \leq \ell \leq \gamma}\left\{L\left(\Gamma_{\ell}\right)\right\} .
$$

Using the fact that, for all $\ell=1, \ldots, \gamma$, the subtree $\Gamma_{\ell}$ contains less than $n$ decomposition subtrees, and applying the induction hypothesis for $\Gamma_{\ell}$ in the first inequality below, gives

$$
\begin{aligned}
\sum_{\mathbf{i} \in \Gamma_{\ell}} \Phi^{s}\left(T_{\mathbf{i}}^{\omega}\right) & \leq M^{\sum_{k=F\left(\Gamma_{\ell}\right)}^{L\left(\Gamma_{\ell}\right)-1}\left(N_{k+1}(\omega)-N_{k}(\omega)\right) \mathbf{1}_{B(\varepsilon)^{c}}\left(\Xi^{k}(\omega)\right)} \\
& \leq M^{\sum_{k=F\left(\Gamma_{\ell_{0}}\right)}^{L\left(\Gamma_{\ell_{\ell}}\right)-1}\left(N_{k+1}(\omega)-N_{k}(\omega)\right) \mathbf{1}_{B(\varepsilon)^{c}}\left(\Xi^{k}(\omega)\right)} .
\end{aligned}
$$

Now there are two cases to consider: $\Gamma(1) \in C(\varepsilon)$ or $\Gamma(1) \in H(\varepsilon, L)$. To begin with, suppose that $\Gamma(1) \in C(\varepsilon)$. Then $\sum_{\mathbf{i} \in \Gamma(1)} \Phi^{s}\left(T_{\mathbf{i}}^{\omega}\right) \leq 1$ and, therefore, by the submultiplicativity of $\Phi^{s}$ and by (3.13), we obtain

$$
\begin{aligned}
& \sum_{\mathbf{i} \in \Gamma} \Phi^{s}\left(T_{\mathbf{i}}^{\omega}\right) \leq \sum_{\ell=1}^{\gamma}\left(\Phi^{s}\left(T_{\mathbf{i}_{\ell}}^{\omega}\right) \sum_{\mathbf{i} \in \Gamma_{\ell}} \Phi^{s}\left(T_{\mathbf{i}}^{\omega}\right)\right) \leq \max _{1 \leq \ell \leq \gamma} \sum_{\mathbf{i} \in \Gamma_{\ell}} \Phi^{s}\left(T_{\mathbf{i}}^{\omega}\right) \\
& \quad \leq M^{\sum_{k=F\left(\Gamma_{\ell_{0}}\right)}^{L\left(\Gamma_{\ell_{1}}\right)-1}\left(N_{k+1}(\omega)-N_{k}(\omega)\right) \mathbf{1}_{B(\varepsilon)^{c}}\left(\Xi^{k}(\omega)\right)} \leq M^{\sum_{k=F(\Gamma)}^{L(\Gamma)-1}\left(N_{k+1}(\omega)-N_{k}(\omega)\right) \mathbf{1}_{B(\varepsilon)^{c}\left(\Xi^{k}(\omega)\right)}} .
\end{aligned}
$$

Hence, (3.12) holds for $\Gamma$.

Finally, assume that $\Gamma(1) \in H(\varepsilon, L)$. Again, by the submultiplicativity of $\Phi^{s}$ and (3.13), we have

$$
\begin{aligned}
\sum_{\mathbf{i} \in \Gamma} \Phi^{s}\left(T_{\mathbf{i}}^{\omega}\right) & \leq \sum_{\ell=1}^{\gamma}\left(\Phi^{s}\left(T_{\mathbf{i}_{\ell}}^{\omega}\right) \sum_{\mathbf{i} \in \Gamma_{\ell}} \Phi^{s}\left(T_{\mathbf{i}}^{\omega}\right)\right) \\
& \leq\left(\sum_{\ell=1}^{\gamma} \Phi^{s}\left(T_{\mathbf{i}_{\ell}}^{\omega}\right)\right) M^{\sum_{k=F\left(\Gamma_{\ell_{0}}\right)}^{L\left(\Gamma_{\ell_{1}}\right)-1}\left(N_{k+1}(\omega)-N_{k}(\omega)\right) \mathbf{1}_{B(\varepsilon)^{c}}\left(\Xi^{k}(\omega)\right)} .
\end{aligned}
$$

Since $\Gamma(1) \in H(\varepsilon, L)$, all the words in $\Gamma(1)$ have the same length and, therefore, $F\left(\Gamma_{\ell_{0}}\right)=L(\Gamma(1))$. Furthermore, $\Xi^{j}(\omega) \notin B(\omega)$ for $j=0, \ldots, L(\Gamma(1))-1$. Hence,

$$
\begin{aligned}
\sum_{\ell=1}^{\gamma} \Phi^{s}\left(T_{\mathbf{i}_{\ell}}^{\omega}\right) & \leq M^{\sum_{k=F(\Gamma(1))}^{L(\Gamma(1))-1}\left(N_{k+1}(\omega)-N_{k}(\omega)\right) \mathbf{1}_{B(\varepsilon)^{c}}\left(\Xi^{k}(\omega)\right)} \\
& =M^{\sum_{k=F(\Gamma)}^{F\left(\Gamma_{\ell_{0}}\right)-1}\left(N_{k+1}(\omega)-N_{k}(\omega)\right) \mathbf{1}_{B(\varepsilon)^{c}}\left(\Xi^{k}(\omega)\right)} .
\end{aligned}
$$

Employing (3.15) in (3.14) and observing that $L\left(\Gamma_{\ell_{1}}\right) \leq L(\Gamma)$, yields

$$
\sum_{\mathbf{i} \in \Gamma} \Phi^{s}\left(T_{\mathbf{i}}^{\omega}\right) \leq M^{\sum_{k=F(\Gamma)}^{L(\Gamma)-1}\left(N_{k+1}(\omega)-N_{k}(\omega)\right) \mathbf{1}_{B(\varepsilon)^{c}}\left(\Xi^{k}(\omega)\right)} .
$$

We conclude that (3.12) is true for $\Gamma$. 
Now we are ready to state and prove our main theorem. We denote the packing and box counting dimensions by $\operatorname{dim}_{\mathrm{P}}$ and $\operatorname{dim}_{\mathrm{B}}$, respectively.

Theorem 3.5. Assume that $\bar{\sigma}<1 / 2$ and $P$ is an ergodic $\Xi$-invariant probability measure on $\Omega$ with $\int_{\Omega} N_{1}(\omega) d P(\omega)<\infty$. Then for P-almost all $\omega \in \Omega$,

$$
\operatorname{dim}_{\mathrm{H}} A_{\mathbf{a}}^{\omega}=\operatorname{dim}_{\mathrm{P}} A_{\mathbf{a}}^{\omega}=\operatorname{dim}_{\mathrm{B}} A_{\mathbf{a}}^{\omega}=\min \left\{s_{0}, d\right\} \text { for } \mathcal{L}^{d \mathcal{A}} \text {-almost all } \mathbf{a} \in \mathbb{R}^{d \mathcal{A}}
$$

where $s_{0}$ is the unique zero of the pressure (see Theorem 2.1).

Proof. Noting that the proof of the upper bound $\operatorname{dim}_{\mathrm{B}} A_{\mathrm{a}}^{\omega} \leq \min \left\{s_{0}, d\right\}$ given in $[21$, Theorem 5.1] is valid under the assumptions of Theorem [3.5, we may restrict our consideration to the Hausdorff dimension. By Theorem 2.3 and Proposition 3.1, it is sufficient to verify that $s_{0}=\alpha$.

Let $s>s_{0}$. By Theorem [2.1, the pressure is strictly decreasing and, therefore, $p^{\omega}(s)<0$ for $P$-almost all $\omega \in \Omega$. Considering such $\omega \in \Omega$ and using the definition of the pressure (2.4), we conclude that $\sum_{\mathbf{i}_{N_{k}(\omega)} \in \Sigma_{*}^{\omega}} \Phi^{s}\left(T_{\mathbf{i}_{N_{k}(\omega)}^{\omega}}\right) \leq 1$ for all sufficiently large $k \in \mathbb{N}$. Recalling Proposition 3.2 , the definition of the neck affinity dimension (3.2) implies that $\alpha=\tilde{\alpha}^{\omega} \leq s$ for $P$-almost all $\omega \in \Omega$. This completes the proof of the fact that $\alpha \leq s_{0}$.

For the purpose of proving the opposite inequality, consider $s>\alpha$ and $\varepsilon>0$. Let $B(\varepsilon) \subset \Omega$ be as in Lemma 3.3. Applying the Birkhoff's ergodic theorem and recalling (3.5), implies that for $P$-almost all $\omega \in \Omega$,

$$
\lim _{n \rightarrow \infty} \frac{1}{N_{n}(\omega)} \sum_{k=0}^{n-1}\left(N_{k+1}(\omega)-N_{k}(\omega)\right) \mathbf{1}_{B(\varepsilon)}\left(\Xi^{k}(\omega)\right)=\frac{\int_{B(\varepsilon)} N_{1}(\omega) d P(\omega)}{\int_{\Omega} N_{1}(\omega) d P(\omega)}=: Q(\varepsilon) .
$$

The fact that $N_{1}(\omega)$ is $P$-integrable, gives $\lim _{\varepsilon \rightarrow 0} Q(\varepsilon)=1$. Let $Q_{L}(\varepsilon)$ be as in Lemma 3.4. Since for $P$-almost all $\omega \in \Omega$,

$$
\lim _{L \rightarrow \infty} \frac{Q_{L}(\varepsilon)}{N_{L}(\omega)}=1-Q(\varepsilon)
$$

and, moreover,

$$
\lim _{L \rightarrow \infty} \frac{N_{L}(\omega)-N_{L-R}(\omega)}{N_{L}(\omega)}=0
$$

by (3.4), Lemma 3.4 implies that for $P$-almost all $\omega \in \Omega$,

$$
p^{\omega}(s)=\lim _{L \rightarrow \infty} \frac{1}{N_{L}(\omega)} \log \sum_{\mathbf{i}_{N_{L}(\omega)} \in \Sigma_{*}^{\omega}} \Phi^{s}\left(T_{\mathbf{i}_{N_{L}}(\omega)}^{\omega}\right) \leq(1-Q(\varepsilon)) \log M \underset{\varepsilon \rightarrow 0}{\longrightarrow} 0 .
$$

From this, we conclude that $s \geq s_{0}$.

Remark 3.6. As pointed out in [21], the upper bound $1 / 2$ for $\bar{\sigma}$ is optimal in Theorem 3.5. Moreover, it is quite natural to assume that the first neck level is integrable. Without the integrability condition it is difficult to utilise the shift invariance and ergodicity of $P$. If $P$ is not ergodic, one may use the ergodic decomposition of $P$ 
and apply Theorem 3.5 to ergodic components of $P$. However, in this case, the dimension may depend on $\omega \in \Omega$. Therefore, the assumptions of Theorem 3.5 may be regarded as optimal ones.

\section{REFERENCES}

[1] M. Barnsley, J. E. Hutchinson and Ö. Stenflo, V-variable fractals: Fractals with partial self similarity, Adv. Math. 218 (2008), 2051-2088.

[2] J. Barral and D.-J. Feng, Multifractal formalism for almost all self-affine measures, Comm. Math. Phys. 318 (2013), 473-504.

[3] T. Bedford, Crinkly curves, Markov partitions and dimension, Ph.D. Thesis, University of Warwick, 1984.

[4] K. Dajani, K. Jiang and T. Kempton, Self-affine sets with positive Lebesgue measure, Indag. Math. (N.S.) 25 (2014), no. 4, 774-784.

[5] K. J. Falconer, The Hausdorff dimension of self-affine fractals, Math. Proc. Cambridge Philos. Soc. 103 (1988), 339-350.

[6] K. J. Falconer, Dimensions of self-affine sets: a survey in Further developments in fractals and related fields, 115-134, Trends Math., Birkhäuser/Springer, New York, 2013.

[7] K. J. Falconer and J. Miao, Dimensions of self-affine fractals and multifractals generated by upper triangular matrices, Fractals 15 (2007), 289-299.

[8] K. J. Falconer and J. Miao, Exceptional sets for self-affine fractals, Math. Proc. Cambridge Philos. Soc. 145 (2008), 669-684.

[9] K. J. Falconer and J. Miao, Random subsets of self-affine fractals, Mathematika 56 (2010), $61-76$.

[10] K. J. Falconer and A. Sloan, Continuity of subadditive pressure for self-affine sets, Real Anal. Exchange 34 (2009), 413-427.

[11] D.--J. Feng, Lyapunov exponents for products of matrices and multifractal analysis, Part II: General matrices, Israel J. Math. 170 (2009), 355-394.

[12] D.-J. Feng and P. Shmerkin, Non-conformal repellers and the continuity of pressure for matrix cocycles, Geom. Funct. Anal. 24 (2014), 1101-1128.

[13] D.-J. Feng and Y. Wang, A class of self-affine sets and self-affine measures, J. Fourier Anal. Appl. 11 (2005), 107-124.

[14] A. Ferguson, T. Jordan and M. Rams, Dimension of self-affine sets with holes, Ann. Acad. Sci. Fenn. Math. 40 (2015), 63-88.

[15] A. Ferguson, T. Jordan and P. Shmerkin, The Hausdorff dimension of the projections of self-affine carpets, Fund. Math. 209 (2010), no. 3, 193-213.

[16] J. Fraser, Dimension theory and fractal constructions based on self-affine carpets, Ph.D. Thesis, University of St. Andrews, 2013.

[17] J. Fraser, Assouad type dimensions and homogeneity of fractals, Trans. Amer. Math. Soc. 366 (2014), no. 12, 6687-6733.

[18] J. Fraser and P. Shmerkin, On the dimensions of a family of overlapping self-affine carpets, Ergodic Theory Dynam. Systems, to appear.

[19] T. Jordan, M. Pollicott and K. Simon, Hausdorff dimension for randomly perturbed self affine attractors, Comm. Math. Phys. 270 (2007), 519-544.

[20] T. Jordan and M. Rams, Packing spectra for Bernoulli measures supported on BedfordMcMullen carpets, Fund. Math. 229 (2015), no. 2, 171-196.

[21] E. Järvenpää, M. Järvenpää, A. Käenmäki, H. Koivusalo, Ö. Stenflo and V. Suomala, Dimensions of random affine code tree fractals, Ergodic Theory Dynam. Systems. 34 (2014), $854-875$. 
[22] E. Järvenpää, M. Järvenpää, B. Li and Ö. Stenflo, Random affine code tree fractals and Falconer-Sloan condition, Ergodic Theory Dynam. Systems, to appear.

[23] R. D. Mauldin and M. Urbański, Graph Directed Markov Systems: Geometry and Dynamics of Limit Sets, Cambridge Tracts in Mathematics 148, Cambridge University Press, Cambridge, 2003.

[24] C. McMullen, The Hausdorff dimension of general Sierpinski carpets, Nagoya Math. J. 96 (1984), 1-9.

[25] F. Przytycki and M. Urbański, On the Hausdorff dimension of some fractal sets, Studia Math. 93 (1989), 155-186.

[26] T. Rajala and M. Vilppolainen, Weakly controlled Moran constructions and iterated function systems in metric spaces, Illinois J. Math. 55 (2011), no. 3, 1015-1051.

[27] E. Rossi, Local dimensions of measures on infinitely generated self-affine sets, J. Math. Anal. Appl. 413 (2014), no. 2, 1030-1039.

[28] K. Simon, The dimension theory of almost self-affine sets and measures, in Fractals, wavelets, and their applications, 103-127, Springer Proc. Math. Stat., 92, Springer, Cham, 2014.

[29] B. Solomyak, Measure and dimensions for some fractal families, Math. Proc. Cambridge Philos. Soc. 124 (1998), 531-546.

Department of Mathematical Sciences, P.O. Box 3000, 90014 University of Oulu, FINLAND

E-mail address: esa.jarvenpaa@oulu.fi

Department of Mathematical Sciences, P.O. Box 3000, 90014 University of Oulu, FINLAND

E-mail address: maarit.jarvenpaa@oulu.fi

Department of Mathematical Sciences, P.O. Box 3000, 90014 University of Oulu, FINLAND

E-mail address: meng.wu@oulu.fi

Department of Mathematical Sciences, P.O. Box 3000, 90014 University of Oulu, FINLAND

School of Mathematics and Statistics, Hubei University, Wuhan 430062, P.R. CHINA

E-mail address: wen.wu@oulu.fi; hust.wuwen@gmail.com 